\title{
Application of Nematophagous Fungi and Salicylic Acid as Biological Control Agents against Root-Knot Nematode, Meloidogyne incognita Infected Gladiolus: A Valuable Export Plant
}

\author{
M.S. Abo-Korah \\ Econ. Entomol. and Agric. Zool. Dept., Fac. Agric., Menoufia Univ., Shibin El-Kom, Egypt.
}

Corresponding author email: abokora_2030@yahoo.com

\begin{abstract}
This study aims to use safety agents to control the root-knot nematode, Meloidogyne incognita infected gladiolus plants (Gladiolus grandiflorus) in pots under field conditions, directly by using two nematophagous fungi, Purpureocillium lilacinum which parasite on nematode eggs and Hirsutella rhossiliensis which parasite on second stage juvenile of nematode, and indirectly by using treatment of salicylic acid (SA). The applied agents applied either individually or in combination, reduced nematode numbers and improved gladiolus vegetative and flowering characteristics. Tri-treatment $(\mathrm{SA}+P$. lilacinum $+H$. rhossiliensis $)$ achieved the best results aptly, leading to increase reduction percentage of nematode juveniles to $80.2 \%$, decreased egg masses production and root gall index to 91.3 and $83.3 \%$, respectively, compared with bionematicide Tervigo (Abamectin) which gave reduction percentage of nematode juveniles as $78.9 \%$, decreased egg masses production and root gall index (80.0, 66.7\%, respectively). Also, the previous tri-treatment improved flowering traits as it led to an increment proportion of flowering stem length $(58.6 \%)$; number of florets/spike (46.2\%) and fresh weight of inflorescence (67.9\%) compared with Tervigo (43.3, 41.8 and 54.2\%, respectively). In addition, tri-treatment (SA $+P$. lilacinum $+H$. rhossiliensis) significantly improved chemical properties of $\mathrm{N}, \mathrm{P}, \mathrm{K}$ and total carbohydrate $(76.8,52.4,47.4$ and $31.4 \%$, respectively) compared with Tervigo (42.7, 23.8, 6.3 and $4.5 \%$, respectively). The obtained results can be concluded that, both nematophagous fungi complement each other, and adding SA led to their superiority in its effectiveness for $M$. incognita control over the nematicide Tervigo (Abamectin). Therefore, the use of chemical nematicide in which causes harmful effects to environment and humans should be reduced or substituted with clean biological control.
\end{abstract}

Keywords: Meloidogyne incognita, biological control, P. lilacinum, H. rhossiliensis, Gladiolus, Abamectin.

\section{INTRODUCTION}

Gladiolus grandiflorus L., is a bulbous plant of great economic value as its multicolored flowers used commercially throughout the world (Mohsin et al., 2015). Gladiolus is considered as one of the best ornamental plants that can achieve a 
significant increase in national income by exporting its high value flowers, as they are highly needed in foreign and domestic markets, especially through the winter season (Sheela, 2008). Gladiolus plants are known to be infected with many pests and pathogens, but the most dangerous is the root-knot nematodes especially Meloidogyne incognita that infect gladiolus roots and cause great economic losses by affecting the appearance of flowers and causing damage to corms and cormles (Khanna and Chandel, 1997). M. incognita is considered an endoparasite and sedentary nematode that causes root knots on gladiolus roots and inflicts great flower damage. However, few studies have been conducted to manage this pest on gladiolus plants (Ravishankar and Singh, 2005).

Salicylic acid, 2-hydroxy benzoic acid (SA), is a phenolic plant hormone that has an important role in regulating water and nutrient absorption as well as regulating plant and flower growth and development (Klessig and Malamy, 1994). Moreover, the use of salicylic acid helps to absorb ions, regulate the rate of photosynthesis and transpiration, as well as improve the activities of antioxidant enzymes and also increase the ability of intentions to withstand abiotic stress (He et al., 2002).

The nematophagous fungus, Purpureocillium lilacinum (Thom) (formely Paecilomyces lilacinus) attacks $M$. incognita eggs and creates an enzymatic defects in the egg shell and eliminate its contents (Khan et al., 2004). It also secretes many enzymes, such as chitinase, serine and protease, which dissolve the egg shell; penetrated and eliminated within it (Fan et al., 2016).

Moreover, the nematophagous fungus, Hirsutella rhossiliensis (Minter) is used as a good and forceful biological enemy to nematode juveniles, the fungus forms adhesive conidia in order to stick to the wall of the nematode juveniles meaning that the mycelium acts prosperous as a sticky trap (Shu et al., 2015).

With the growing environmental awareness and recognition of the dangers of chemical pesticides that have been used for more than fifty years in controlling many pests, many pesticides have lost their effectiveness, and most pests have acquired the characteristic of pesticide resistance, and many organisms such as fish, birds, pollinators, flowers and mammals have been significantly damaged. Hence, the aim of the present research came to appliance clean control, by studying the antagonistic properties of nematophagous fungi, Purpureocillium lilacinum and Hirsutella rhossiliensis as well as salicylic acid against M.incognita infected gladiolus plants compared with Tervigo (Abamectin a natural fermentation product of Streptomyces avermitilis).

\section{MATERIALS AND METHODS}

Potted experiment was conducted under field conditions at the experimental farm of the Faculty of Agriculture, Menoufia University, Shebin El-Kom , Egypt to shed light on the impact of three treatments, salicylic acid (SA); Purpureocillium lilacinum and Hirsutella rhossiliensis, either single; dual or triad, in comparison with the biocide, Tervigo (Abamectin) for controlling the root knot nematode Meloidogyne incognita infected gladiolus plants and its connectedness with plants flowering; vegetative and chemical characteristics.

\section{Nematode culture}

M. incognita juveniles $\left(\mathrm{J}_{2 \mathrm{~s}}\right)$ were collected from pure culture on cotton Gossypium barbadense (Gallini) variety Delta Pin61. The cotton roots with egg masses were washed in the water to clean them from soil sticking to the roots, then the roots were cut to pieces of $1 \mathrm{~cm}$ long and stirred in sodium hypochlorite $(0.5 \%)$ 
for 3 minutes and shake well in sterile water ( Kerry and Bourne, 2002) to free eggs from egg masses. Eggs were incubated at $25 \pm 1^{\circ} \mathrm{C}$ for 3 to 4 days to obtain second stage juveniles $\left(\mathrm{J}_{2 \mathrm{~s}}\right)$ with the aid of modified Baermann funnel method (Gray, 1984) and used in the experimental infection procedure (Chuixu et al., 2013).

\section{Preparation of fungal inoculum}

Isolates of the two nematophagus fungi; $H$. rhossiliensis and $P$. lilacinum were obtained from Cairo Mercin, Ain Shams University, Egypt. These isolates were separately cultured on PDA (Potato Dextrose Agar), and then each one was added aseptically as mycelial mat to a flask containing $50 \mathrm{~g}$ of autoclaved wheat grains and incubated at $25{ }^{\circ} \mathrm{C}$ for seven days. Subcultures of the obtained isolates were kept on PDA slants and stored at $5^{\circ} \mathrm{C}$ until used.

\section{Experimental preparation and design}

A randomized design with four replicates was used, gladiolus corms were imported from Holland with size of $(8-10 \mathrm{~cm}$ in circumference) and planted in PVC pots $(30 \mathrm{~cm}$ in diameter). Each pot was filled with $4 \mathrm{~kg}$ of sterilized clay-sand mixed soil $(1: 1, \mathrm{v} / \mathrm{v})$, one week after planting. Gladiolus corms were infected with second stage juveniles $\left(\mathrm{J}_{2 \mathrm{~s}}\right)$ of $M$. incognita nematode which induced at a rate $1000 \mathrm{~J}_{2} /$ pot / corm and added by pipette into three holes around corm. Simultaneously, the two nematophagous fungi, $H$. rhossiliensis and $P$. lilacinum (grown on wheat grains) were introduced to the soil at the rate of $3 \mathrm{~g} / \mathrm{pot} / \mathrm{corm}$ of wheat grains infected with each fungus. Foliar application of SA $(150 \mathrm{ppm})$ was adopted three weeks after planting and then monthly in the early morning till the end of the experiment (Hussien and Moussa, 2018).

Nematicide Tervigo ${ }^{\mathrm{TM}}$ is a suspension concentrate (SC) containing 20g/L abamectin with the addition of an iron chelate Fe-EDDHA $400 \mathrm{~g} / \mathrm{L}$ produced by Syngenta East Africa Ltd. Abamectin as an active ingredient provides aptly control of nematodes. After one week of seedlings adaptation, Tervigo was applied as $0.3 \mathrm{ml}$ per pot as soil drench around the corm.

\section{Nematode Extraction and Enumeration}

Three months after the beginning of the experiment, plant roots were submerged, thoroughly washed in tap water, immediately stained with acid fuchsin in lactophenol and stored in it for not less than 24 hours. Plant roots were rinsed in water, root knot nematode females were counted with the help of a dissecting microscope and number of galls rated as mentioned in Table (1).

Table 1: Rating scale levels of galls numbers (Taylor \&Sasser 1978)

\begin{tabular}{cc}
\hline Number of galls/ root system & Gall index \\
\hline 0 & 0 \\
$1-2$ & 1 \\
$3-10$ & 2 \\
$11-30$ & 3 \\
$31-100$ & 4 \\
$>100$ & 5 \\
\hline
\end{tabular}


Egg masses were assessed by staining the roots with Phloxin-B solution $(0.15 \mathrm{~g} / \mathrm{l}$ liter tap water) for 20 minutes according to Daykin and Hussey (1985). Each composite soil sample was carefully mixed, and an aliquot of $100 \mathrm{~cm} 3$ was processed for nematode extraction according to methods described by Southey (1970). Each treatment consists of four replicates, and samples were taken periodically after 4,8 , 12 and 16 weeks, then the flowering, vegetative and chemical characteristics were recorded at the end of experiment.

\section{Chemical analysis}

Total carbohydrate percentages were measured in the aerial parts of gladiolus plants by using the colorimetric method (Dubios et al., 1956). Nitrogen, phosphorus and potassium (NPK) percentages were determined in the dried herb by Kjeldah method, Spectrophotometericly and Flame photometer methods respectively, according to Cottenie et al. (1982).

\section{Statistical analysis}

The obtained data were subjected to analysis of variance (ANOVA) using Costat Software, Version 6.4 (2008). The mean differences were compared by Least Significant Difference (L.S.D. 5\%)

Reduction percentages were computed according to Abbott formula (1925):

Increase or decrease $\%=$ Control - Treatment $/$ Control x 100

\section{RESULTS AND DISCUSSION}

Data in Table (2) indicate that all treatments decreased the numbers of root knot nematode, Meloidogyne incognita juveniles with varying proportions, and significant differences were observed among all treatments. Triple treatment $(\mathrm{SA}+P$. lilacinum $+H$. rhossiliensis) gave the highest nematode mortality rate $(80.2 \%)$, followed by a nematicide Tervigo (78.9\%). The dual treatment ( P. lilacinum $+H$. rhossiliensis) gave a death rate of $70.6 \%$ which is higher than that occurred by each fungus separately i.e. P. lilacinum $(58.9 \%)$ and $H$. rhossiliensis $(65.7 \%)$, and this indicates that both fungi complemented each other in its effectiveness on nematode and there is no contradiction between them. These results coincide with those obtained by Khairy et al. (2019) and Paul et al. (2019) who stated that P. lilacinum destroy root-knot nematode eggs and reduce the number of juveniles $\left(\mathrm{J}_{2}\right)$ by $52 \%$ and this fungus penetrates the egg shell and causes an enzymatic and physiological defect resulting from the formation of compounds toxic to nematodes.

The obtained results in Table (3) show that, triple treatment $(\mathrm{SA}+$ P. lilacinum + $H$. rhossiliensis) gave the highest percentages of deficiency in mature females; egg masses and root gall index at a rate of $87.4 ; 91.3$ and $83.3 \%$, respectively, while the nematicide Tervigo gave $83.3 ; 80.0$ and $66.7 \%$, respectively. $H$. rhossiliensis an endoparasite which produces sticky materials on the surface of mycelium to catch nematode prey at any point on the spinning surface; reduce cyst nematode eggs and larvae by $95 \%$ under greenhouse condition (Hallmann et al., 2018; Wang et al., 2007) as well as reduce number of M.incognita juveniles in soil, root galling and egg masses 
( Mostafa, 1998; Shu et al., 2015). On the other hand, Abamectin is widely used as nematicide on root- knot nematode (Muzhandu et al., 2014 ; Beixing et al., 2018).

Table 2: Impact of treatments adopted singly or solidarity on the population density of root-knot nematode, Meloidogyne incognita infesting gladiolus plants and reduction $\%$, under field conditions.

\begin{tabular}{|c|c|c|c|c|c|c|c|c|c|c|}
\hline \multirow{3}{*}{ Treatments } & \multicolumn{5}{|c|}{$\begin{array}{c}\text { Aver. no. of Meloidogyne incognita } \\
\text { juveniles/ } 100 \mathrm{~g} \text { soil }\end{array}$} & \multirow{2}{*}{\multicolumn{5}{|c|}{ Reduction \% }} \\
\hline & \multicolumn{5}{|c|}{ weeks after treatments } & & & & & \\
\hline & $\begin{array}{c}4 \\
\text { week }\end{array}$ & $\begin{array}{c}8 \\
\text { week }\end{array}$ & $\begin{array}{c}12 \\
\text { week }\end{array}$ & $\begin{array}{c}16 \\
\text { week }\end{array}$ & $\begin{array}{c}\text { Over } \\
\text { all } \\
\text { mean }\end{array}$ & $\begin{array}{c}4 \\
\text { wee } \\
k\end{array}$ & $\begin{array}{c}8 \\
\text { week }\end{array}$ & $\begin{array}{c}12 \\
\text { week }\end{array}$ & $\begin{array}{c}16 \\
\text { week }\end{array}$ & $\begin{array}{c}\text { Over } \\
\text { all } \\
\text { mean }\end{array}$ \\
\hline $\begin{array}{l}\text { Salicylic acid } \\
\text { (SA) }\end{array}$ & $\begin{array}{c}917 \\
\mathrm{~B}\end{array}$ & $\begin{array}{c}893 \\
\text { B }\end{array}$ & $\begin{array}{c}816 \\
\text { B }\end{array}$ & $\begin{array}{c}789 \\
\mathrm{~b}\end{array}$ & $\begin{array}{c}853.8 \\
\mathrm{~b}\end{array}$ & 33.4 & 44.2 & 55.5 & 62.8 & 48.9 \\
\hline P. lilacinum & $\begin{array}{r}902 \\
\mathrm{C} \\
\end{array}$ & $\begin{array}{c}763 \\
\mathrm{C} \\
\end{array}$ & $\begin{array}{c}512 \\
\mathrm{C} \\
\end{array}$ & $\begin{array}{c}491 \\
\mathrm{c}\end{array}$ & $\begin{array}{c}667.1 \\
\mathrm{c}\end{array}$ & 34.4 & 52.4 & 72.1 & 76.8 & 58.9 \\
\hline H. rhossiliensis & $\begin{array}{c}803 \\
\mathrm{E}\end{array}$ & $\begin{array}{c}594 \\
\mathrm{E}\end{array}$ & $\begin{array}{c}431 \\
\mathrm{E}\end{array}$ & $\begin{array}{c}390 \\
\mathrm{~d}\end{array}$ & $\begin{array}{c}554.5 \\
\mathrm{e}\end{array}$ & 41.6 & 62.9 & 76.5 & 81.6 & 65.7 \\
\hline $\mathrm{SA}+$ P.lilacinum & $\begin{array}{c}826 \\
D\end{array}$ & $\begin{array}{c}701 \\
\mathrm{D}\end{array}$ & $\begin{array}{c}492 \\
\mathrm{D}\end{array}$ & $\begin{array}{c}374 \\
\mathrm{e}\end{array}$ & $\begin{array}{c}598.3 \\
\mathrm{~d}\end{array}$ & 39.9 & 56.2 & 73.2 & 82.4 & 62.9 \\
\hline $\begin{array}{l}\mathrm{SA}+H . \\
\text { rhossiliensis }\end{array}$ & $\begin{array}{c}764 \\
F\end{array}$ & $\begin{array}{c}509 \\
\mathrm{~F}\end{array}$ & $\begin{array}{c}397 \\
\mathrm{~F}\end{array}$ & $\begin{array}{c}316 \\
\mathrm{f}\end{array}$ & $\begin{array}{l}496.5 \\
\mathrm{f}\end{array}$ & 44.5 & 68.2 & 78.4 & 85.1 & 69.1 \\
\hline $\begin{array}{l}\text { P.lilacinum }+H \\
\text {.rhossiliensis }\end{array}$ & $\begin{array}{c}704 \\
G\end{array}$ & $\begin{array}{c}493 \\
G\end{array}$ & $\begin{array}{c}373 \\
G\end{array}$ & $\begin{array}{c}294 \\
\mathrm{~g}\end{array}$ & $\begin{array}{c}466.0 \\
\mathrm{~g}\end{array}$ & 48.8 & 69.2 & 79.7 & 86.1 & 70.6 \\
\hline $\begin{array}{l}\mathrm{SA}+P . \text { lilacinum }+ \\
\text { H. rhossiliensis }\end{array}$ & $\begin{array}{c}583 \\
\mathrm{H}\end{array}$ & $\begin{array}{c}340 \\
\mathrm{I}\end{array}$ & $\begin{array}{c}196 \\
\text { I }\end{array}$ & $\begin{array}{c}105 \\
\mathrm{I}\end{array}$ & $\begin{array}{l}306.0 \\
\mathrm{i}\end{array}$ & 57.6 & 78.8 & 89.3 & 95.0 & 80.2 \\
\hline $\begin{array}{l}\text { Tervigo } \\
\text { ( Abamectin) }\end{array}$ & $\begin{array}{c}531 \\
\mathrm{I}\end{array}$ & $\begin{array}{c}394 \\
\mathrm{H}\end{array}$ & $\begin{array}{c}223 \\
\mathrm{~h}\end{array}$ & $\begin{array}{c}186 \\
\mathrm{~h}\end{array}$ & $\begin{array}{c}333.5 \\
\mathrm{~h}\end{array}$ & 61.4 & 75.4 & 87.8 & 91.2 & 78.9 \\
\hline Control & $\begin{array}{c}1376 \\
\mathrm{~A} \\
\end{array}$ & $\begin{array}{c}1602 \\
\mathrm{~A} \\
\end{array}$ & $\begin{array}{c}1834 \\
\mathrm{a} \\
\end{array}$ & $\begin{array}{c}2119 \\
\mathrm{a} \\
\end{array}$ & $\begin{array}{c}1732.8 \\
\mathrm{a} \\
\end{array}$ & - & - & - & - & - \\
\hline LSD 5\% & 8.6 & 8.5 & 8.6 & 9.2 & 8.5 & - & - & - & - & - \\
\hline
\end{tabular}

Means in each column followed by the same letter (s) are not significantly different at $5 \%$ level.

The obtained results in Table (4) characterize that, adding foliar application of SA either with $P$. lilacinum or $H$. rhossiliensis fungi in the dual treatment, resulted improvements in growth characters than that found when apply each fungus adopted separately. Triple treatment $(\mathrm{SA}+P$. lilacinum $+H$. rhossiliensis $)$ accomplished best results to take into increments the flowering stem length; number of florets/spike; fresh weigh of inflorescence and fresh weight of root with percentages of +58.6; $+46.2 ;+67.9$ and $+97.3 \%$, respectively, superior to that found by nematicide Tervigo, which gave less percentage $(+43.3 ;+41.8 ;+54.2$ and $+74.7 \%$, respectively). The importance of addition of SA foliar application appears in its effect by improving the mineral content NPK and total carbohydrates of gladiolus plants (Table 5), especially when its additional to the triad treatment $(\mathrm{SA}+P$. lilacinum $+H$. rhossiliensis $)$, which led to increase of these chemical properties in proportions reaching $+76.8 ;+52.4$; +47.4 and $+31.4 \%$, respectively superior to the dual treatment $(P$. lilacinum $+H$. 
rhossiliensis) which gave $+40.8 ;+23.8 ;+9.5$ and $+6.9 \%$, respectively and advanced also over Tervigo which gave percentages $+42.7 ;+23.8 ;+6.0$ and $4.5 \%$, respectively.

Table 3: Influence of adopted treatments on Meloidogyne incognita mature females; egg masses and root gall index.

\begin{tabular}{|c|c|c|c|c|c|c|}
\hline \multirow[b]{2}{*}{ Treatments } & \multirow{2}{*}{$\begin{array}{l}\text { Mature } \\
\text { females } \\
/ 1 \mathrm{~g} \text { root }\end{array}$} & \multirow{2}{*}{$\begin{array}{l}\text { No } \\
\text { Egg } \\
\text { masses }\end{array}$} & \multirow[b]{2}{*}{$\begin{array}{l}\text { Root gall } \\
\text { index }\end{array}$} & \multicolumn{3}{|c|}{ Decrease $\%$} \\
\hline & & & & Females & $\begin{array}{c}\text { Egg } \\
\text { masses }\end{array}$ & $\begin{array}{l}\text { Root gall } \\
\text { index }\end{array}$ \\
\hline \multirow{2}{*}{ Slicylic acid (SA) } & 15.2 & 34.9 & 4.0 & \multirow{2}{*}{-36.4} & \multirow{2}{*}{-25.9} & \multirow{2}{*}{-20.0} \\
\hline & B & $\mathrm{b}$ & $\mathrm{b}$ & & & \\
\hline \multirow{2}{*}{ P. lilacinum } & 11.0 & 18.1 & 3.0 & \multirow{2}{*}{-53.9} & \multirow{2}{*}{-61.6} & \multirow{2}{*}{-40.0} \\
\hline & $\mathrm{C}$ & d & $\mathrm{c}$ & & & \\
\hline \multirow{2}{*}{ H. rhossiliensis } & 9.8 & 24.3 & 3.0 & \multirow{2}{*}{-58.9} & \multirow{2}{*}{-48.4} & \multirow{2}{*}{-40.0} \\
\hline & $\mathrm{Cd}$ & $\mathrm{c}$ & $\mathrm{c}$ & & & \\
\hline \multirow{2}{*}{$\mathrm{SA}+P$. lilacinum } & 8.1 & 13.8 & 2.0 & \multirow{2}{*}{-66.1} & \multirow{2}{*}{-70.7} & \multirow{2}{*}{-60.0} \\
\hline & De & e & d & & & \\
\hline \multirow{2}{*}{$\mathrm{SA}+H$. rhossiliensis } & 7.5 & 17.7 & 2.0 & \multirow{2}{*}{-68.6} & \multirow{2}{*}{-62.4} & \multirow{2}{*}{-60.0} \\
\hline & Ef & d & d & & & \\
\hline \multirow{2}{*}{$\begin{array}{l}\text { P. lilacinum }+H \text {. } \\
\text { rhossiliensis }\end{array}$} & 6.0 & 11.0 & 2.0 & \multirow{2}{*}{-74.9} & \multirow{2}{*}{-76.6} & \multirow{2}{*}{-60.0} \\
\hline & $\mathrm{F}$ & ef & d & & & \\
\hline $\mathrm{SA}+P$. lilacinum + & 3.0 & 4.1 & 1.0 & \multirow{2}{*}{-87.4} & \multirow{2}{*}{-91.3} & \multirow{2}{*}{-80.0} \\
\hline H. rhossiliensis & G & $\mathrm{g}$ & $\mathrm{e}$ & & & \\
\hline Tervigo & 4.0 & 9.4 & 2.0 & \multirow{2}{*}{-83.3} & \multirow{2}{*}{-80.0} & \multirow{2}{*}{-60.0} \\
\hline (Abamectin) & G & $\mathrm{f}$ & $d$ & & & \\
\hline Control & 23.9 & 47.1 & 5.0 & 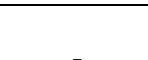 & 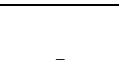 & 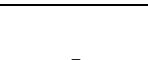 \\
\hline COIIIU⿴囗十 & A & $\mathrm{a}$ & A & - & - & - \\
\hline LSD 5\% & 1.7 & 3.4 & 0.2 & - & - & - \\
\hline
\end{tabular}

Means in each column followed by the same letter (s) are not significantly different at 5\% level.

The most enhancement effective in this regard was obtained by using SA (150 $\mathrm{ppm})$. The promotive effect of SA on growth characters could be attributed to its bioregulator effects on physiological and biochemical processes in plants such as ion uptake; cell elongation; cell division; cell differentiation; sink and source regulation; enzymatic activities; protein synthesis and photosynthetic activity; as well as; increase the antioxidant capacity of plant. SA as ant stress substance which enhance the plant tolerance to environmental stresses. 
Table 4: Effect of different treatments on some growth characters of gladiolus infected with Meloidogyne incognita.

\begin{tabular}{|c|c|c|c|c|c|c|c|c|}
\hline \multirow[b]{2}{*}{ Treatments } & \multirow[b]{2}{*}{$\begin{array}{c}\text { Flowering } \\
\text { stem } \\
\text { length } \\
(\mathrm{cm})\end{array}$} & \multirow[b]{2}{*}{$\begin{array}{l}\text { Number } \\
\text { florets } \\
\text { /spike }\end{array}$} & \multirow{2}{*}{$\begin{array}{l}\text { Fresh } \\
\text { weight } \\
\text { inflores } \\
\text { cence } \\
\text { (g) }\end{array}$} & \multirow[b]{2}{*}{$\begin{array}{l}\text { Fresh } \\
\text { weight } \\
\text { root } \\
(\mathrm{g})\end{array}$} & \multicolumn{4}{|c|}{ Increase $\%$} \\
\hline & & & & & $\begin{array}{c}\text { Flowering } \\
\text { stem } \\
\text { length }\end{array}$ & $\begin{array}{l}\text { Number } \\
\text { florets } \\
\text { /spike }\end{array}$ & $\begin{array}{l}\text { Fresh } \\
\text { weight } \\
\text { inflores } \\
\text { cence }\end{array}$ & $\begin{array}{c}\text { Fresh } \\
\text { weight } \\
\text { root }\end{array}$ \\
\hline \multirow{2}{*}{ Salicylic acid (SA) } & 59.5 & 9.8 & 29.5 & 9.0 & \multirow{2}{*}{+18.3} & \multirow{2}{*}{+7.7} & \multirow[b]{2}{*}{+16.6} & \multirow{2}{*}{+20.0} \\
\hline & $\mathrm{F}$ & $\mathrm{H}$ & $\mathrm{e}$ & $\mathrm{h}$ & & & & \\
\hline \multirow{2}{*}{ P. lilacinum } & 62.0 & 10.0 & 32.3 & 10.1 & \multirow{2}{*}{+23.3} & \multirow{2}{*}{+9.9} & \multirow{2}{*}{+27.7} & \multirow{2}{*}{+34.7} \\
\hline & $\mathrm{Ef}$ & $\mathrm{G}$ & $\mathrm{De}$ & $\mathrm{g}$ & & & & \\
\hline \multirow{2}{*}{ H.rhossiliensis } & 65.2 & 10.5 & 33.9 & 10.5 & \multirow{2}{*}{+29.6} & \multirow{2}{*}{+15.4} & \multirow{2}{*}{+33.9} & \multirow{2}{*}{+40.0} \\
\hline & De & $\mathrm{F}$ & de & $\mathrm{f}$ & & & & \\
\hline \multirow{2}{*}{$\mathrm{SA}+$ P.lilacinum } & 67.0 & 11.2 & 34.5 & 11.3 & \multirow{2}{*}{+33.2} & \multirow{2}{*}{+23.1} & \multirow{2}{*}{+36.4} & \multirow{2}{*}{+50.7} \\
\hline & $\mathrm{Cd}$ & $\mathrm{E}$ & $\mathrm{cd}$ & $\mathrm{e}$ & & & & \\
\hline \multirow{2}{*}{$\mathrm{SA}+$ H.rhossiliensis } & 69.3 & 12.0 & 36.8 & 12.0 & \multirow{2}{*}{+37.8} & \multirow{2}{*}{+31.8} & \multirow{2}{*}{+45.5} & \multirow{2}{*}{+60.0} \\
\hline & $\mathrm{Bc}$ & $\mathrm{D}$ & $\mathrm{bc}$ & $\mathrm{d}$ & & & & \\
\hline \multirow{2}{*}{$\begin{array}{l}\text { P. lilacinum }+ \\
\text { H.rhossiliensis }\end{array}$} & 70.2 & 12.5 & 37.0 & 12.8 & \multirow{2}{*}{+39.6} & \multirow{2}{*}{+37.4} & +462 & +707 \\
\hline & $\mathrm{Bc}$ & $\mathrm{C}$ & $\mathrm{bc}$ & $\mathrm{c}$ & & & & $+/ 0.1$ \\
\hline $\mathrm{SA}+P$. lilacinum & 79.8 & 13.3 & 42.5 & 14.8 & & 460 & 670 & 073 \\
\hline$+H$. rhossiliensis & A & A & $\mathrm{a}$ & $\mathrm{a}$ & +58.6 & +46.2 & $+6 / .9$ & +97.3 \\
\hline Tervigo & 72.1 & 12.9 & 39.0 & 13.1 & & & & \\
\hline (Abamectin) & $\mathrm{B}$ & $\mathrm{B}$ & $\mathrm{ab}$ & $\mathrm{b}$ & +43 & +41.8 & +54.2 & +74.7 \\
\hline & 50.3 & 9.1 & 25.3 & 7.5 & 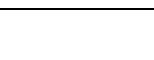 & - & 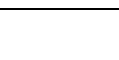 & - \\
\hline Control & G & I & $\mathrm{f}$ & $\mathrm{i}$ & - & - & - & \\
\hline LSD $5 \%$ & 3.4 & 0.2 & 3.8 & 0.2 & - & - & - & - \\
\hline
\end{tabular}

Means in each column followed by the same letter (s) are not significantly different at 5\% level.

These results are in quite agreement with the findings of Hussien and Moussa, (2018); Al-Qubaie (2013) on Jasminum sambac and Ali et al. (2016) on Ammi visnaga. It has long been known that salicylic acid (SA) increases plant resistance in so-called acquired systemic resistance (Sergio et al., 2014). Recently, Khairy et al. (2021) studied the possibility of managing $M$. incognita infecting eggplant using moringa extracts, vermicompost, and two commercial bio-products, and resulted promising road.

It could be concluded that, integration several organisms can be an exquisitely model for nematode control which gave implicitly and explicitly prosperity in its aptly and could be forceful effectively used with other cultural, biological and chemical nematode management strategies. 
Table 5: Effect of different treatments on NPK and total carbohydrate contents of gladiolus plants infected with Meloidogyne incognita.

\begin{tabular}{|c|c|c|c|c|c|c|c|c|}
\hline \multirow[b]{2}{*}{ Treatments } & \multirow[b]{2}{*}{$\mathrm{N}(\%)$} & \multirow[b]{2}{*}{$\mathrm{P}(\%)$} & \multirow[b]{2}{*}{$\mathrm{K}(\%)$} & \multirow[b]{2}{*}{$\begin{array}{c}\text { Total } \\
\text { carbo. } \\
(\%)\end{array}$} & \multicolumn{4}{|c|}{ Increase $\%$} \\
\hline & & & & & $\begin{array}{l}\mathrm{N} \\
(\%)\end{array}$ & $\begin{array}{l}\mathrm{P} \\
(\%)\end{array}$ & $\begin{array}{c}\mathrm{K} \\
(\%)\end{array}$ & $\begin{array}{c}\text { Total } \\
\text { carbo. } \\
(\%)\end{array}$ \\
\hline Salicylic acid (SA) & $\begin{array}{c}3.11 \\
\mathrm{~cd}\end{array}$ & $\begin{array}{c}0.28 \\
\mathrm{bc}\end{array}$ & $\begin{array}{c}1.43 \\
\mathrm{C}\end{array}$ & $\begin{array}{c}12.14 \\
\mathrm{D}\end{array}$ & +47.4 & +33.3 & +23.4 & +15.6 \\
\hline P. lilacinum & $\begin{array}{c}2.71 \\
\mathrm{f}\end{array}$ & $\begin{array}{c}0.24 \\
\mathrm{f}\end{array}$ & $\begin{array}{c}1.21 \\
\mathrm{E}\end{array}$ & $\begin{array}{c}10.86 \\
\mathrm{H}\end{array}$ & +28.4 & +14.3 & +4.3 & +3.4 \\
\hline H. rhossiliensis & $\begin{array}{c}2.92 \\
\mathrm{e}\end{array}$ & $\begin{array}{c}0.25 \\
\text { ef }\end{array}$ & $\begin{array}{c}1.23 \\
\mathrm{E}\end{array}$ & $\begin{array}{c}11.13 \\
F\end{array}$ & +38.4 & +16.0 & +6.0 & +6.0 \\
\hline $\mathrm{SA}+$ P.lilacinum & $\begin{array}{c}3.24 \\
\text { bc }\end{array}$ & $\begin{array}{c}0.27 \\
\mathrm{~cd}\end{array}$ & $\begin{array}{c}1.54 \\
\mathrm{~B}\end{array}$ & $\begin{array}{c}12.86 \\
\mathrm{C}\end{array}$ & +53.6 & +28.6 & +32.8 & +22.5 \\
\hline $\begin{array}{l}\text { SA+ } \\
\text { H.rhossiliensis }\end{array}$ & $\begin{array}{c}3.41 \\
\mathrm{~b}\end{array}$ & $\begin{array}{c}0.29 \\
b\end{array}$ & $\begin{array}{c}1.56 \\
\mathrm{~B}\end{array}$ & $\begin{array}{c}12.97 \\
\text { B }\end{array}$ & +61.6 & +38.1 & +34.5 & +23.5 \\
\hline $\begin{array}{l}\text { P. lilacinum }+H . \\
\text { rhossiliensis }\end{array}$ & $\begin{array}{c}2.97 \\
\mathrm{de}\end{array}$ & $\begin{array}{c}0.26 \\
\text { de }\end{array}$ & $\begin{array}{c}1.27 \\
\mathrm{D}\end{array}$ & $\begin{array}{c}11.23 \\
\mathrm{E}\end{array}$ & +40.8 & +23.8 & +9.5 & +6.9 \\
\hline $\begin{array}{l}\mathrm{SA}+P . \text { lilacinum } \\
+H . \text { rhossiliensis }\end{array}$ & $\begin{array}{c}3.73 \\
\mathrm{a} \\
\end{array}$ & $\begin{array}{c}0.32 \\
\mathrm{a}\end{array}$ & $\begin{array}{c}1.71 \\
\mathrm{~A}\end{array}$ & $\begin{array}{c}13.80 \\
\mathrm{~A}\end{array}$ & +76.8 & +52.4 & +47.4 & +31.4 \\
\hline $\begin{array}{l}\text { Tervigo } \\
\text { (Abamectin) }\end{array}$ & $\begin{array}{c}3.01 \\
\mathrm{de}\end{array}$ & $\begin{array}{c}0.26 \\
\text { de }\end{array}$ & $\begin{array}{c}1.23 \\
\mathrm{E} \\
\end{array}$ & $\begin{array}{c}10.97 \\
\mathrm{G}\end{array}$ & +42.7 & +23.8 & +6.0 & +4.5 \\
\hline Control & $\begin{array}{c}2.11 \\
\mathrm{~g}\end{array}$ & $\begin{array}{c}0.21 \\
\mathrm{~g}\end{array}$ & $\begin{array}{c}1.16 \\
\mathrm{~F} \\
\end{array}$ & $\begin{array}{c}10.50 \\
\mathrm{I}\end{array}$ & - & - & - & - \\
\hline LSD $5 \%$ & 0.2 & 0.02 & 0.03 & 0.09 & - & - & - & - \\
\hline
\end{tabular}

Means in each column followed by the same letter (s) are not significantly different at $5 \%$ level.

\section{REFERENCES}

Abbott, W.S. (1925). A method of computing the effectiveness of an insecticide. J. Econ. Entomol. 18: 265-267.

Ali, A. F.; Hassan, E. A.; Hamad, E. H. A. and Ahmed, A. A. M. (2016). Growth and productivity of Ammi visnaga as affected by organic fertilizers rate and antioxidants level. Middle East J. Agric. Res. 5(4): 620-628.

Al-Qubaie, A.I. (2013). Response Jasminum sambac Ait plants to spraying salicylic acid. Met., Env. \& Arid Land Agric. Sci. 24(1): 67-73.

Beixing, L.; Yupeng, R.; Da-xia, Z.; Shuangyu, X.; Wei, M. and Feng, L. (2018) Modifying the formulation of Abamectin to promote its efficacy on southern root-knot nematode (Meloidogyne incognita) under blending-of-soil and rootirrigation conditions. J. Agric. Food Chem. 66 (4):799-805.

Chuixu, K.; Chongyan, Z.; Jing, L.; Jun, Z.; Keqin, Z. and Yajun, L. (2013) Evaluation of Stropharia sp. 1.2052 nematicidal effects against Meloidogyne incognita on tomato. African J. Microbiol. Res. 7(50): 5737-5741.

Cottenie, A.; Verloo, M.; Kiekens, L.; Velghe, G.; and Camerlynck, R. (1982).Chemical analysis of plant and soil.PP. Laboratory of Analytical and Agro chemistry, State.Univ. Ghent. Belgium.3;111-116.

CoStat software program (Version 6.4). CoStat version 6.400 Copyright (C-1998-2008 Cohort Software.798 Lighthouse Ave. PMB 320, Monterey,CA, 93940, USA.

Daykin, M. E. and Hussey, R.S. (1985). Staining and histopathological techniques in nematology. In: Barker, K. R.; C. C. Carter and J. N. Sasser (eds), An 
Advanced treatise on Meloidogyne, Vo. II Methodology, 39-48. North Carolina State University Graphics, Raleigh.

Dubois, M.; Gilles, K.; Hamilton, J.; Robers P.; and Smith, F. (1956).Calorimetric method for determination of sugars and related substance. Anal. Chem. 28; 350-356.

Fan, Y.; Abdelnabby H. and Yan Nong, X. (2016) Construction and verification of a gene knockout system in the nematophagous fungus, Purpureocillium lilacinum (Hypocreales: Ophiocordycipitaceae) Appl. Entomol. Zool. 51: 99110.

Gray, N.F. (1984). Ecology of nematophagous fungi: Comparison of the soil sprinkling method with the Baermann funnel technique in the isolation of endoparasites. Soil Biol. Biochem.16:81-83.

Hallmann, J.; Gutberlet, V.; Klaus, D. J. and Vorlop, D. (2018) Effect of additives on the efficacy of microencapsulated Hirsutella rhossiliensis controlling Heterodera schachtii on sugar beets, J. Biol. Control. 128: 5-9.

He, Y.L.; Liu, Y.L.; Chen, Q. and Bian, A.H. (2002). Thermo tolerance related to antioxidation induced by salicylic acid and heat hardening in tall fescue seedlings. J. Plant Physiol. Mol. Biol. 28: 89-95.

Hussien, E. A. and Moussa, M.M. (2018) Considerable prosperous in successional changes on Foeniculum vulgare, Mill, vegetative growth properties and yield parameters resultant salicylic acid and soil salinity application. Menoufia J. Plant Prot. 2: 7-11.

Kerry, B. and Bourne, J. (2002). A manual for research on Verticillium chlamydosporium, a potential biological control agent for root-knot nematodes. Druckform Gmbhmerckstr, Germany. p .171.

Khairy, Doaa; Refaei, A.R.; Mostafa, Fatma, A.M. (2021). Management of Meloidogyne incognita infecting eggplant using moringa extracts, vermicompost, and two commercial bio-products. Egypt. J. Agronematol.20 (1): 1-16.

Khan, A.; Willianms, K. and Nevalainen, H. (2004) Effects of Paecilomyces lilacinus protease and chitinase on the egg shell structures and hatching of Meloidogyne javanica juveniles. Biol. Control 31:346-352.

Khanna, A.S. and Chandel, S.S. (1997). Damage potential of Meloidogyne incognita in Gladiolus cv. Sylvia. Indian J. Nematol. 27: 253-254.

Klessig, D.F.and Malamy, J. (1994). The salicylic acid signal in plants. Plant Mol. Biol. 26: 1439-1458.

Kumar, D. and Singh, K. (2006). Assessment of predacity and efficacy of Arthrobotrys dactyloides for biological control of root knot disease of tomato. J. Phytopathol. 154:1-5.

Mohsin, B.; Imran, K.; Rashid, W. K.; Mohsin, T.; Muhammad, Z. and Iftikhar, A. (2015). Growth and corm production of Gladiolus grandiflorus L. 'Essential' under different NPK regimes. J. Ornam.Plants 6(1): 11-19.

Mostafa, F.A. (1998). Impact of three nematophagous fungi separately and in combination with poultry manure on root-knot nematode infecting sunflower. Egypt. J. Agronematol. 2(2): 245-256.

Muzhandu, R.; Chinheya C.; Dimbi S. and Manjeru P. (2014) Efficacy of Abamectin for the control of root knot nematodes in tobacco seedling production. African J. Agric. Res. 9(1), 144-147.

Paul, D; Reinhard, E.; Erika, C.; Jürgen, K. and Sebastian, K. (2019). Integrated control of Meloidogyne incognita in tomatoes using fluopyram and 
Purpureocillium lilacinum strain 251. Crop Prot. 124:1-7.

Ravishankar, M. and Singh, R.V. (2005). Effect of carbosulfan (25 STD) and neem seed kernel powder as corm dressing for the management of root-knot nematode (Meloidogyne incognita) infecting gladiolus. Indian J. Nematol. 35(1): 53-55.

Sergio, M.; Elena, F. and Paola, L. (2014) Expression of tomato salicylic acid (SA)responsiv pathogenesis-related genes in Mi-1-mediated and SA-induced resistance to root-knot nematodes. Molecular Plant Pathol. 15(3) :255-264.

Sheela, V.L. (2008). Flowers for trade. New India Publishing Agency, New Delhi, India 254 (2): 65-78.

Shu, C.; Lai Y.; Yang, E.; Chen S.; Xiang, M. and Liu, X. (2015). Functional response of the fungus Hirsutella rhossiliensis to the nematode, Heterodera glycines. Life Sci. J. 58 (7): 704-712.

Southey, J. F. (1970). Laboratory methods for work with plant and soil nematodes. Ministry of Agriculture, Fishers and Food. Technical Bulletin 2:5th ed.,148 pp.

Taylor, A.L.and Sasser, J.A. (1978). Biology, Identification and Control of Root-knot Nematodes (Meloidogyne spp.). Coop Publ. Dep. Plant Pathology North Carolina, State University and US. Agency Int. Dev. Raleigh, NC.111 pp.

Wang, B.; Wenping, W. and Liu, X. (2007) Purification and characterization of a neutral serine protease with nematicidal activity from Hirsutella rhossiliensis. Mycopathologia. 163:169-176. 


$$
\text { الملخص العربي }
$$

\title{
تطبيق الفطريات المضادة وحمض السلسيلك كمواد مكافحة حيوية لنيماتودا تعقد الجذور Meloidogyne incognita
}

\author{
محمد سعيد ابوقورة \\ قسم الحشر ات الاقتصادية و الحيو ان الزر اعى ـ كلية الزر اعة - جامعة المنوفية - مصر
}

تهدف هذه الدراسة لاستخدام طريقة آمنة لمكافحة نيماتودا تعقد الجذور

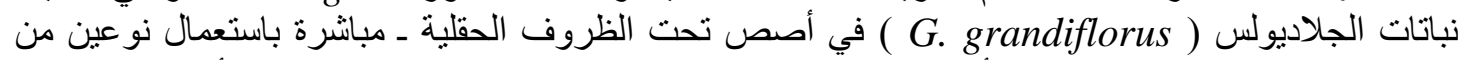

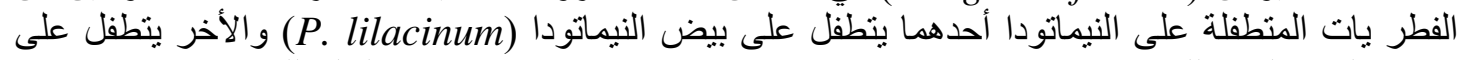

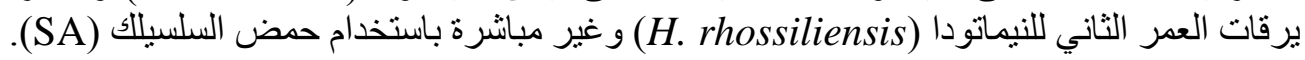

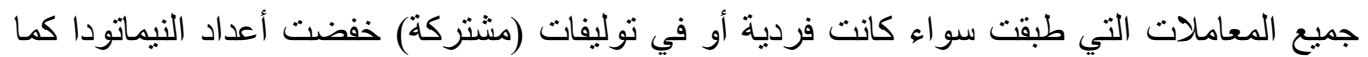

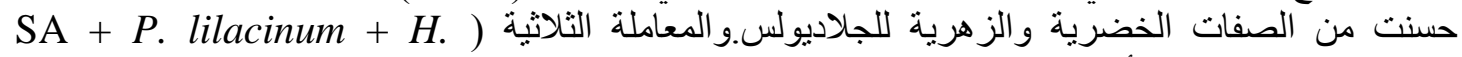
(rhossiliensis

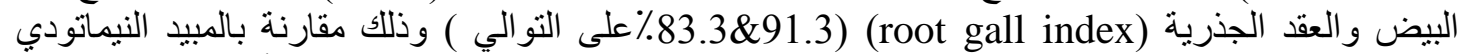

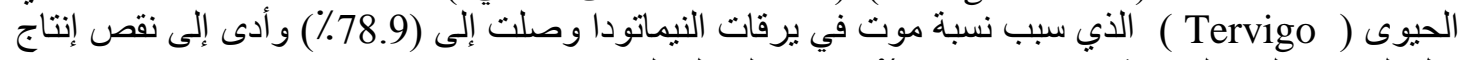

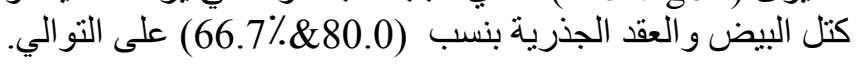

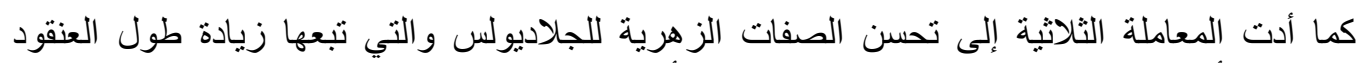

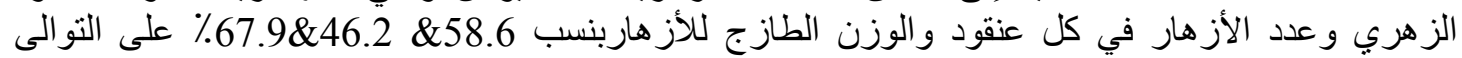

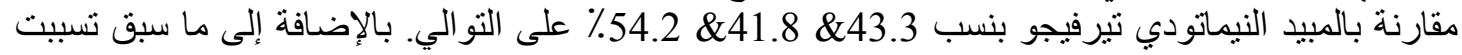

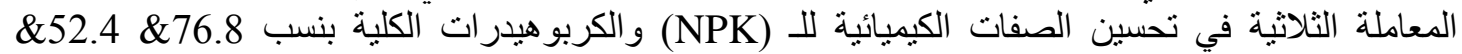

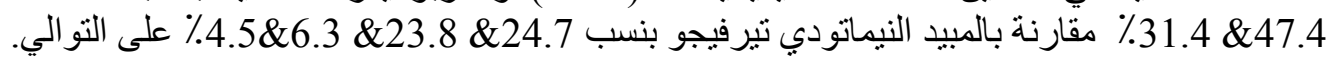

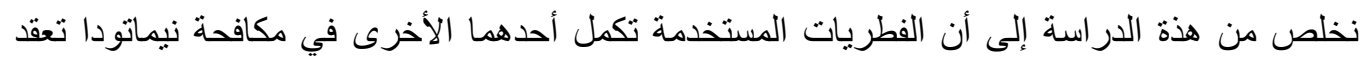

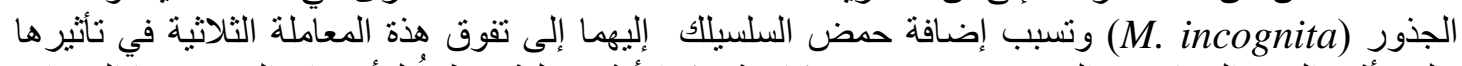

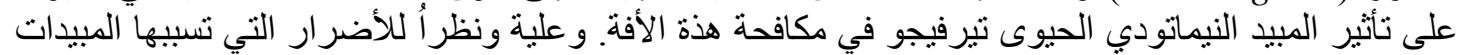

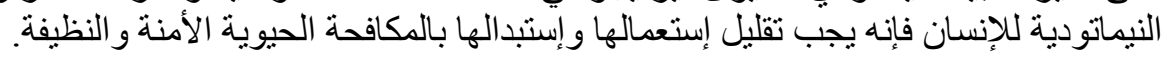

